\title{
Effects of Prior Exposure to Animate Objects on Approach Tendency in Chicks
}

\author{
SUSAN SAEGERT and D. W. RAJECKI ${ }^{1}$ \\ Department of Psychology, The University of Michigan, \\ Ann Arbor, Michigan 48104
}

\begin{abstract}
Earlier research has shown that brief amounts of social stimulation prior to testing results in an increased tendency in chicks to approach and follow a novel object. Those studies could not, however, distinguish between the effects of viewing animate objects and the effects of simple activation due to disturbances in the subjects' rearing situations. The current study manipulated the effects of environmental novelty and exposure to objects independently. It was found that exposure to a novel environment prior to testing produced evidence for activation. Exposure to objects during pronounced changes in the rearing condition reduced latency to approach a novel object in a later test, but exposure to a novel environment without exposure to objects, or exposure to objects under conditions of low environmental novelty, did not produce such a reduction.
\end{abstract}

There is ample evidence that chicks tend to approach familiar animals or objects. Socially reared chicks make reliable social discriminations between broodmates of familiar and unfamiliar color (Rajecki and Lake, 1972; Salzen and Cornell, 1968) and between broodmates and animals of another species (Pattie, 1936). Preference for the familiar also obtains when chicks are imprinted on artificial targets and then tested for discriminative social choices between original and new targets (Guiton, 1959; Sluckin and Taylor, 1964; Smith and Hoyes, 1961).

Conversely, chicks that have been reared socially for prolonged periods appear to be inhibited in responding positively to the exclusive presentation of a novel target. Salzen (1962) reports that chicks reared in isolation for $24 \mathrm{hr}$ were more likely to show pleasure ("contentment" calls, approach, and/or nestling behavior) on the initial presentation of an artificial target (64\% of the group) than were chicks that had been reared socially for the same period (24\% of the group). Guiton (1959) and Sluckin and Salzen (1961) have reported similar findings.

${ }^{1}$ This research was supported by the National Science Foundation Grant NSF GS 3119 to Robert B. Zajonc. 
In light of these findings on the result of prolonged exposure, the additional finding that brief social exposure enhances responsiveness to novel targets is an interesting one. Chicks that had been placed among a group of 10 other chicks for $2 \mathrm{hr}$ immediately prior to a test were more responsive to a blue ball than subjects that had had no prior social experience (Polt and Hess, 1966). Similarly, Graves and Siegel (1968) report that $3 \mathrm{~min}$ of visual stimulation by a human hand just prior to exposure to a complex stimulus array produced more responsive chicks than those that had been maintained in the dark until the test. Recently, we discovered that differential experience with the opening of the incubator door affected chicks' latencies to approach a bank of tiny flashing lights. The data showed a significant linear decline in latency to approach over position in the order of running (Rajecki, Eichenbaum, and Heilweil ${ }^{2}$ ).

Quite probably chicks receiving brief amounts of stimulation prior to testing do not show inhibition of responsiveness toward novel stimuli because strong social bonds with the original stimuli have not been established. The question remains, however, why do they domonstrate increased responsiveness relative to controls? Polt and Hess (1966) suggest that during the original social experience the subjects learn that there are no harmful consequences associated with social objects, hence they are less fearful than controls. Graves and Siegel (1968) suggest that something in the pretest exposure (visual flicker, perhaps) matches something in the test for responsiveness with the result that any response to the original object generalizes to the novel. On the other hand, it is known that chicks treated with adrenergic stimulants show approach tendency superior to controls (Kovach, 1964; Rajecki and Saegert, 1971) as do chicks receiving optimal levels of electric shock (Kovach and Hess, 1963), exposure to loud noises (Pitz and Ross, 1961), or an opportunity to imprint at temperatures colder than the one to which they had been adapted (Fischer, 1970). It is generally held that these treatments increase the arousal level of the subjects in question and that increased arousal leads to increase in approach tendency. A more parsimonious interpretation of the effects of brief prior stimulation may be that it merely has consequences for the general level of activation of the subject and that this activation carries over to the test for approach tendency.

The purpose of the present research was to examine further the effect of brief prior experience on tendency in chicks to approach a novel target. An attempt was made to distinguish between the effects of viewing one or more animate objects and the effect of mere activation stemming from being placed in a novel environment just before testing.

Subjects were 54 DeKalb White Leghorn chicks. Single animals were placed in individual $9.75 \times 7 \times 7$ in. Holtege metal cages lined with black

${ }^{2}$ Rajecki, D. W., Eichenbaum, H., and Heilweil, M. Effects of temperature on vocalizations and imprinting in White Leghorn chicks (unpublished data). 
paper approximately a half hour after hatching. The cages were illuminated and heated to about $95^{\circ} \mathrm{F}$ by overhead lamps. At $16 \mathrm{hr}$ after hatching each subject was removed from its home cage and placed in an identical cage in an exposure apparatus. The wire mesh wall of the cage was butted against a $7 \times 7$ in. opening fitted with a guillotine door. With the door open the cage looked out on a $9.5 \times 6$ in. deep illuminated stage with a floor flush with the bottom of the cage.

While in the apparatus the subjects were given one of six treatments in a $2 \times 3$ experimental design. Half the subjects were assigned to a low "environmental novelty" (low EN) condition: illumination and temperature in the chamber were identical to that of the home cage. The remaining subjects were assigned to a high "environmental novelty" (high EN) condition: the temperature in the chamber was $75^{\circ} \mathrm{F}$ and illumination was absent. Across conditions there were three levels of exposure to moving objects: (a) exposure to no objects, (b) exposure to one object, a red barbell consisting of 2 ping pong balls and a 2 in. rod, and (c) exposure to three objects; the barbell, a 2 in. green cube, and a $2 \times 3$ in. drum with alternating red and green stripes. The objects, presented in the stage area of the apparatus, rotated at $30 \mathrm{rpm}$ on a horizontal axle that was parallel to the wire mesh wall of the exposure cage. The axle was powered by an electric motor that produced a 75-dB hum, measured at the center of the cage.

Exposure consisted of three 3-min periods separated by two 25 -sec interexposure intervals. During the intervals the guillotine door was closed. In the single object condition, the barbell was presented each time. In the multiple object condition, the objects were presented in random sequence. In the no-object condition, nothing was presented but the opening and closing of the door. The sound of the motor was absent.

When the exposure sequence was completed, the subject was returned to its rearing cage for $1 \mathrm{hr}$. At $17 \mathrm{hr}$ of age the subjects were tested for tendency to approach a novel target in the runway described by Rajecki and Saegert (1971). Briefly, an illuminated cage at one end of a 60-in. alley contained a rotating $3 \times 5$ in. yellow card while a cage at the other end was empty. The card rotated at $60 \mathrm{rpm}$ on a horizontal axle parallel to the long axis of the alley and was powered by a motor that produced a 57-dB hum measured 6 in. from the source. The chick was placed in the center of the runway and observed for $10 \mathrm{~min}$. Each chick was assigned a latency score of the minute block during which it crossed a threshold 20 in. from the target cage wall. If the chick failed to cross the threshold it was assigned a maximum latency of $10 \mathrm{~min}$. If a chick crossed a 20 -in. threshold toward the empty cage it was also assigned the maximum latency score. The latency to moving from the start area for such negative responders was noted, however.

In addition to the experimental groups, an extraexperimental comparison group was tested for tendency to approach the yellow card. Subjects 


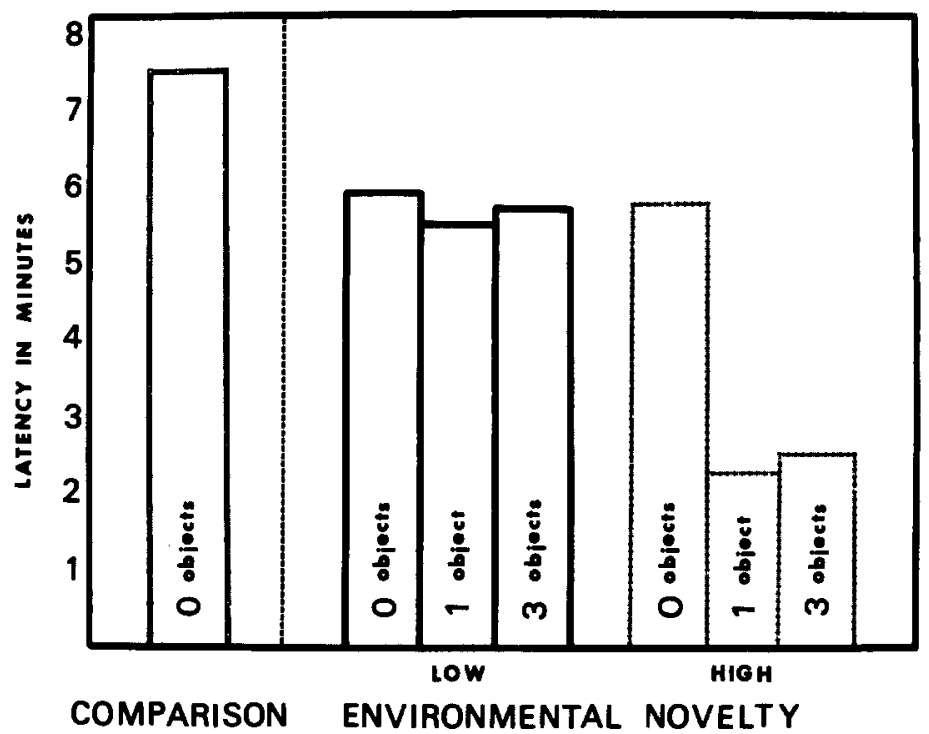

Fig. 1. Average latency in minutes to crossing a threshold toward a novel object by chicks exposed to 0,1 , or 3 objects under various levels of environmental novelty.

in this group were tested at $17 \mathrm{hr}$ of age and were not removed from the rearing cage prior to the test.

The average time in minutes to crossing the approach threshold is shown in Fig. 1. Analysis of variance of the latency scores showed that the chicks in the high EN group approached the target more quickly than those in the low EN group $(F=(1,48) 5.68, p<.025)$. There was no main effect for the level of exposure to objects $(F=1.91$, ns) nor was there an interaction effect $(F=1.28$, ns). However, it is clear in Fig. 1 that there are sharp differences between the birds exposed to some objects and those exposed to none in the high EN group. This difference is substantiated by an analysis of variance of simple effects of levels of exposure over levels of environmental novelty: there is no effect for objects in the low EN group ( $F<1$, ns) but there is a respectable effect for objects in the high EN group $(F=(2,48) 3.15$, $p<.053)$.

Comparisons between the three main groups provide additional information about the effects of exposure to a novel environment. When latency to movement either toward or away from the card was examined it was found that the high EN group (mean $=2.78 \mathrm{~min}$ ) differed significantly from the comparison group (mean $=7.56 \mathrm{~min}$ ) in a direction consistent with the notion that disturbance produces activation $(t=(34) 5.27, p<.005)$. General activation was also suggested by the difference between the low EN group $($ mean $=5.22 \mathrm{~min})$ and the comparison group $(t=(34) 1.87, p<.05)$. The two groups exposed to different levels of environmental novelty were also different from one another $(t=(52) 3.15, p<.005)$. 
The data suggest that exposure to a novel environment prior to testing increases activation in chicks. Marked increases in activation, however, did not increase tendency to approach the target unless stimulation by animate objects had also been provided during that period. These results are consistent with the report of Polt and Hess (1964) that, for 16-hr old, dark-reared chicks, $2 \mathrm{hr}$ of light experience prior to testing had less effect on approach tendency than did $2 \mathrm{hr}$ of social experience. The current findings support the conclusions of Polt and Hess (1966) and Graves and Siegel (1968) that specific brief visual or social stimulation is a factor in enhancing the tendency in chicks to approach a novel target, in addition to the effects of simple activation by changes in the environment. ${ }^{3}$ Exposure to objects under conditions of low environmental novelty does not appear to be a sufficient condition to enhance approach tendency to a novel object.

It has been suggested to the authors that the enhancement of approach tendency in chicks as a result of prior exposure to animate objects reported in this study may be a marginal effect that obtained only because of the relatively unattractive or impoverished nature of the novel target employed. That is, if a biologically appropriate or otherwise attractive novel target were to be employed the effects of prior exposure might be overwhelmed. It should be recalled in this connection that Polt and Hess (1966) employed a moving object painted blue, a color attractive to chicks (see Salzen, Lily, and McKeown, 1971), that emitted an intermittent auditory signal. Moreover, Graves and Siegel (1968) used a target that consisted of flashing lights, an oscillating pendulum, and a continuous recording of the broody call of a hen. In both studies, of course, effects of prior exposure emerged. These findings belie the suggestion that prior exposure effects may be limited to chicks tested with an impoverished novel target.

\section{REFERENCES}

Fischer, G. J. (1970) Arousal and impairment: Temperature effects on following during imprinting. J. Comp. Physiol. Psychol. 73, 412-420.

Graves, H. B., and Siegel, P. B. (1968) Prior experience and the approach response in domestic chicks. Anim. Behav. 16, 18-23.

Guiton, P. (1959) Socialization and imprinting in Brown Leghorn chicks. Anim. Behav. 7, 26-34.

Kovach, J. K. (1964) Effects of autonomic drugs on imprinting. J. Comp. Physiol. Psychol. 57 183-187.

Kovach, J. K., and Hess, E. H. (1963) Imprinting: Effects of painful stimulation upon the following response. J. Comp. Physiol. Psychol. 56, 461-464.

${ }^{3}$ It should be noted that in the high EN, 0 object group the relatively long mean latency of $5.78 \mathrm{~min}$ was produced in part by four chicks that approached the empty cage and were assigned maximum approach latencies. For the five positive responders in the group the mean latency of approach to the target was $2.40 \mathrm{~min}$. 
Pattie, F. A. Jr. (1936) The gregarious behavior of normal chicks and chicks hatched in isolation. J. Comp. Psychol. 21, 161-178.

Pitz, G. F., and Ross, R. B. (1961) Imprinting as a function of arousal. J. Comp. Physiol. Psychol. 54, 602-604.

Polt, J. M., and Hess, E. H. (1964) Effects of light and social experience on following and imprinting. Science 143, 1185-1187.

Polt, J. M., and Hess, E. H. (1966) Effects of social experience on the following response in chicks. J. Comp. Physiol. Psychol. 61, 268-270.

Rajecki, D. W., and Lake, D. (1972) Social preference in chicks as a function of own color and rearing condition. Rev. Comp. Animal 6, 151-156.

Rajecki, D. W., and Saegert, S. (1971) Effects of methamphetamine hydrochloride on imprinting in White Leghorn chicks. Psychon. Sci. 23, 7-8.

Salzen, E. A. (1962) Imprinting and fear. Symp. Zoo. Soc. London 8, 199-217.

Salzen, E. A., and Cornell, J. M. (1968) Self-perception and species recognition in birds. Behaviour 30, 44-65.

Salzen, E. A., Lily, R. E., and McKeown, J. R. (1971) Colour preference and imprinting in domestic chicks. Anim. Behav. 19, 542-547.

Sluckin, W., and Salzen, E. A. (1961) Imprinting and perceptual learning. Quart. J. Exp. Psychol. 13, 65-77.

Sluckin, W., and Taylor, K. F. (1964) Imprinting and short-term retention. Brit $J$. Psychol. 55, 181-187.

Smith, F. V., and Hoyes, P. A. (1961) Properties of the visual stimuli for the approach response in the domestic chick. Anim. Behav. 9, 159-166. 